Appl. Set-Valued Anal. Optim. 1 (2019), No. 3, pp. 337-347

Available online at http://asvao.biemdas.com

https://doi.org/10.23952/asvao.1.2019.3.07

\title{
THE SUCCESSIVE CONTRACTION METHOD FOR FIXED POINTS OF PSEUDO-CONTRACTIVE MAPPINGS
}

\author{
CAIPING YANG, SONGNIAN HE*
}

College of Science, Civil Aviation University of China, Tianjin 300300, China

\begin{abstract}
In this paper, a new general iterative method is proposed for finding a fixed point of a Lipschitz continuous pseudo-contractive mapping defined on a closed convex subset of a real Hilbert space. Since each iteration of our method can be reduced to finding the fixed point of a strict contraction, our iterative method is called the successive contraction method. We give exact and inexact versions of the successive contraction method and prove their weak convergence, respectively. The main advantage of our method is that its convergence does not require the compactness assumption on the operators under consideration, which is quite different from the existing algorithms such as the Ishikawa iterative method. This superiority is also demonstrated by comparing the numerical performances of our method and the Ishikawa iteration process.

Keywords. Successive contraction method; Pseudo-contractive mapping; Fixed point; Lipschitz continuous; Weak convergence.
\end{abstract}

2010 Mathematics Subject Classification. 47H09, 65K10.

\section{INTRODUCTION}

Let $\mathscr{H}$ be a real Hilbert space with inner product $\langle\cdot, \cdot\rangle$ and induced norm $\|\cdot\|$. Given a nonempty closed convex set $C \subset \mathscr{H}$ and a mapping $T: C \rightarrow C, T$ is said to be

(i) monotone if

$$
\langle T(x)-T(y), x-y\rangle \geq 0, \quad \forall x, y \in C ;
$$

(ii) pseudo-contractive if

$$
\| T\left(x-T(y)\left\|^{2} \leq\right\| x-y\left\|^{2}+\right\|(I-T)(x)-(I-T)(y) \|^{2}, \quad \forall x, y \in C ;\right.
$$

(iii) $L$-Lipschitz continuous if there exists a constant $L>0$ such that

$$
\|T(x)-T(y)\| \leq L\|x-y\|, \quad \forall x, y \in C ;
$$

(iv) nonexpansive if

$$
\|T(x)-T(y)\| \leq\|x-y\|, \quad \forall x, y \in C
$$

${ }^{*}$ Corresponding author.

E-mail addresses: cpyang@cauc.edu.cn (C. Yang), songnianhe@163.com (S. He).

Received May 25, 2019; Accepted September 17, 2019.

(C)2019 Applied Set-Valued Analysis and Optimization 
Notice that (1.2) can be rewritten as

$$
\langle x-y, T(x)-T(y)\rangle \leq\|x-y\|^{2}, \quad \forall x, y \in C .
$$

Comparing (1.3) with (1.4), it is easy to see that the nonexpansive mapping class is a true subclass of the pseudocontractive mapping class. We use $\operatorname{Fix}(T):=\{x \in C \mid T x=x\}$ to denote the fixed point set of $T$.

Because many nonlinear problems, such as, convex optimization problems, can be finalized to zero point problems of monotone operators, the research on the iterative algorithms for finding zero points of monotone operators has attracted much attention; see, e.g., $[2,3]$ and the references therein. From (1.4), we know that $T$ is pseudo-contractive if and only if its complement operator $I-T$ is monotone. Based on this fact, it is not difficult to see that zero point problems of monotone operators are equivalent to fixed point problems of pseudo-contractive mappings. For this reason, many authors focused on iterative methods for fixed points of pseudocontractive mappings during the past decade, see, for example, $[1,4,5,6,13,17,19]$ and the references therein. Especially, great attention has been paid to nonexpansive mappings (a special kind of pseudo-contractive mappings) because many nonlinear problems can be reduced to fixed point problems of nonexpansive mappings, see, for example, [8, 9, 10, 11, 12, 15, 16] and the references therein. Among these iterative methods, the Mann iteration method is the most favour fixed point algorithm for nonexpansive mappings since many algorithms can be reduced to Mann iteration [2]. Recall that Mann's iteration process [15] is defined as follows:

$$
\left\{\begin{array}{l}
x^{0} \in C \text { chosen arbitrarily, } \\
x^{n+1}=\alpha_{n} x^{n}+\left(1-\alpha_{n}\right) T x^{n}, n \geq 0,
\end{array}\right.
$$

where $\left\{\alpha_{n}\right\}_{n=0}^{\infty}$ is in $[0,1]$. One of the fundamental convergence results on the Mann iterative method is the following.

Theorem 1.1. ([2, Theorem 5.14]) Let $C$ be a nonempty closed convex subset of a real Hilbert space $\mathscr{H}$ and let $T: C \rightarrow C$ be a nonexpansive mapping such that $F i x(T) \neq \emptyset$. Let $\left\{\alpha_{n}\right\}_{n=0}^{\infty}$ be a sequence in $[0,1]$ such that

$$
\sum_{n=0}^{+\infty} \alpha_{n}\left(1-\alpha_{n}\right)=\infty
$$

Then $\left\{x^{n}\right\}_{n=0}^{\infty}$ generated by (1.5) converges weakly to a fixed point of $T$.

In 1974, to find a fixed point of a Lipschitz continuous pseudo-contractive mapping $T: C \rightarrow$ $C$, Ishikawa [13] introduced the following iteration process:

$$
\left\{\begin{array}{l}
x^{0} \in C \text { chosen arbitrarily, } \\
y^{n}=\left(1-\alpha_{n}\right) x^{n}+\alpha_{n} T x^{n}, n \geq 0, \\
x^{n+1}=\left(1-\beta_{n}\right) x^{n}+\beta_{n} T y^{n}, n \geq 0,
\end{array}\right.
$$

where $\left\{\alpha_{n}\right\}_{n=0}^{\infty}$ and $\left\{\beta_{n}\right\}_{n=0}^{\infty}$ are real sequences in $[0,1]$. In some sense, (1.7) is more general than the Mann iteration process, which is now referred to as Ishikawa iterative method. Ishikawa proved the following convergence result.

Theorem 1.2. [13] Let $C$ be a compact convex subset of a real Hilbert space $\mathscr{H}$ and let $T$ : $C \rightarrow C$ be a Lipschitz continuous pseudocontractive mapping such that Fix $(T) \neq \emptyset$. If $\left\{\alpha_{n}\right\}_{n=0}^{\infty}$ 
and $\left\{\beta_{n}\right\}_{n=0}^{\infty}$ satisfy the conditions:

$$
\text { (i) } 0 \leq \beta_{n} \leq \alpha_{n} \leq 1 \text {; (ii) } \lim _{n \rightarrow \infty} \alpha_{n}=0 \text {; (iii) } \sum_{n=0}^{\infty} \alpha_{n} \beta_{n}=\infty,
$$

then the sequence $\left\{x^{n}\right\}_{n=0}^{\infty}$ generated by scheme (1.7) converges strongly to a fixed point of T.

The Ishikawa iterative method (1.7) has been studied extensively by many authors, see [7, 19, 20]. However it remains an open question whether or not this method can be employed to approximate fixed points of Lipschitz pseudocontractive mappings without the compactness assumption on $C$ or $T$.

In this paper, a new general iterative method is proposed for finding a fixed point of a Lipschitz continuous pseudo-contractive mapping $T: C \rightarrow C$. Since each iteration of our algorithm is reduced to finding the fixed point of a strict contraction, it is called the successive contraction method. We give exact and inexact versions of the successive contraction method and prove their weak convergence respectively. The main advantage of our method is that its convergence does not require the compactness assumption on $C$ or $T$, which is quite different from the existing algorithms such as the Ishikawa iterative method. This superiority is also demonstrated by comparing the numerical performances of our method and the Ishikawa iteration process in solving an example.

\section{PRELIMINARIES}

In this section, we recall some basic concepts and conclusions that will be used in this paper. In the rest of this paper, we always use $\mathscr{H}$ to indicate a real Hilbert space and use $I$ to denote the identity operator on $\mathscr{H}$. Also, we will use the following notations:

(i) $\rightarrow$ denotes strong convergence.

(ii) $\rightarrow$ denotes weak convergence.

(iii) $\omega_{w}\left(x_{n}\right)=\left\{x \mid \exists\left\{x_{n_{k}}\right\}_{k=1}^{\infty} \subset\left\{x_{n}\right\}_{n=1}^{\infty}\right.$ such that $\left.x_{n_{k}} \rightarrow x\right\}$ denotes the weak $\omega$-lim set of $\left\{x_{n}\right\}_{n=1}^{\infty}$.

The following conclusion can be easily verified.

Lemma 2.1. Let $C \subset \mathscr{H}$ be a nonempty closed convex set and let $T: C \rightarrow C$ be a mapping. Then the following statements are equivalent.

(i) $T$ is pseudo-contractive;

(ii) $\langle x-y, T(x)-T(y)\rangle \leq\|x-y\|^{2}, \forall x, y \in C$;

(iii) $I-T$ is monotone.

Lemma 2.2. [16] Let $T: C \rightarrow C$ be a nonexpansive mapping. Then $I-T$ is demiclosed at 0 in the sense that if $\left\{x^{n}\right\}_{n=0}^{\infty}$ is a sequence in $C$ such that $x^{n} \rightarrow x$ and $\left\|x^{n}-T x^{n}\right\| \rightarrow 0$, as $n \rightarrow \infty$, then $x-T x=0$, i.e., $x \in F i x(T)$.

Lemma 2.3. [18] Assume that $\left\{a_{n}\right\}_{n=0}^{\infty},\left\{\lambda_{n}\right\}_{n=0}^{\infty}$ and $\left\{\sigma_{n}\right\}_{n=0}^{\infty}$ are three sequences of nonnegative real numbers such that

$$
a_{n+1} \leq\left(1+\lambda_{n}\right) a_{n}+\sigma_{n}, \quad \forall n \geq 0 .
$$

If $\sum_{n=0}^{\infty} \lambda_{n}<+\infty$ and $\sum_{n=0}^{\infty} \sigma_{n}<+\infty$, then $\lim _{n \rightarrow \infty} a_{n}$ exists. 
Lemma 2.4. [14] Assume that $\left\{a_{n}\right\}_{n=0}^{\infty}$ is a sequence of nonnegative real numbers such that

$$
a_{n+1} \leq\left(1-\lambda_{n}\right) a_{n}+\lambda_{n} \delta_{n}, n \geq 0,
$$

where $\left\{\lambda_{n}\right\}_{n=0}^{\infty}$ is a sequence in $(0,1)$ and $\left\{\delta_{n}\right\}_{n=0}^{\infty}$ is a real sequence such that

(i) $\sum_{n=0}^{\infty} \lambda_{n}=\infty$;

(ii) $\limsup _{n \rightarrow \infty} \delta_{n} \leq 0$ or $\sum_{n=0}^{\infty}\left|\lambda_{n} \delta_{n}\right|<\infty$.

Then $\lim _{n \rightarrow \infty} a_{n}=0$.

Recall that a function $\varphi: C \rightarrow \mathbb{R}$ is said to be lower semi-continuous (lsc) at $u \in C$ if $\left\{u^{n}\right\}_{n=0}^{\infty} \subset C$ satisfying $u_{n} \rightarrow u$ implies

$$
\varphi(u) \leq \liminf _{n \rightarrow \infty} \varphi\left(u^{n}\right) .
$$

Recall that $\varphi: C \rightarrow \mathbb{R}$ is said to be weakly lower semi-continuous (w-lsc) at $u \in C$ if $\left\{u^{n}\right\}_{n=0}^{\infty} \subset$ $C$ satisfying $u_{n} \rightarrow u$ implies

$$
\varphi(u) \leq \liminf _{n \rightarrow \infty} \varphi\left(u^{n}\right) .
$$

It is well known that if $\mathscr{H}$ is finite dimensional, then the lower semi-continuity is consistent with the weaker lower semi-continuity.

\section{THE EXACT SUCCESSIVE CONTRACTION METHOD}

Let $C$ be a nonempty closed convex subset of $\mathscr{H}$ and let $T: C \rightarrow C$ be a $L$-Lipschitz continuous pseudo-contractive mapping with a nonempty fixed point set, i.e., Fix $(T):=\{x \in C \mid T(x)=$ $x\} \neq \emptyset$. In this section, we propose the exact successive contraction method to find an element in Fix $(T)$. Each iteration of our algorithm simply boils down to calculating the unique fixed point of a strict contraction.

Algorithm 3.1. (The exact successive contraction method)

Step 1: Choose $x^{0} \in C$ arbitrarily and set $n:=0$.

Step 2: For the current $x^{n}$, choose $\alpha_{n}$ such that $0<\alpha_{n}<\min \left\{1, \frac{1}{L}\right\}$, set $T_{n}: C \rightarrow C$ by

$$
T_{n}(x):=\left(1-\alpha_{n}\right) x^{n}+\alpha_{n} T(x), \forall x \in C,
$$

and find $\bar{x}^{n+1}$ such that

$$
\bar{x}^{n+1}=T_{n} \bar{x}^{n+1} \text {. }
$$

Step 3: Set

$$
x^{n+1}=\bar{x}^{n+1},
$$

$n:=n+1$ and return to Step 2.

Remark 3.1. For each $n \geq 0$, due to $0<\alpha_{n}<\frac{1}{L}$, it is easy to see that $T_{n}$ is a strict contraction with the coefficient $\gamma_{n}=\alpha_{n} L$. Hence each iteration of Algorithm 3.1 simply boils down to calculating the unique fixed point of a strict contraction. This is why Algorithm 3.1 is called the 
successive contraction method. The subproblem (3.2) is easy to solve. In fact, Banach contraction mapping principle suggests that the unique fixed point $\bar{x}^{n+1}$ of $T_{n}$ can be approximated by the sequence $\left\{x_{m}^{n+1}\right\}_{m=0}^{\infty}$ generated by the scheme:

$$
\left\{\begin{array}{l}
x_{0}^{n+1} \in C \text { chosen arbitrarily } \\
x_{m+1}^{n+1}=T_{n}\left(x_{m}^{n+1}\right), m \geq 0
\end{array}\right.
$$

Also, there exists the error estimate

$$
\left\|x_{m}^{n+1}-\bar{x}^{n+1}\right\| \leq \frac{\gamma_{n}^{m}}{1-\gamma_{n}}\left\|x_{1}^{n+1}-x_{0}^{n+1}\right\|, \quad m \geq 1 .
$$

Theorem 3.1. Let $C$ be a nonempty closed convex subset of $\mathscr{H}$ and let $T: C \rightarrow C$ be a $L$ Lipschitz continuous pseudo-contractive mapping. If $\|\cdot-T(\cdot)\|: C \rightarrow \mathbb{R}^{1}$ is a weakly lower semi-continuous function, and $\left\{\alpha_{n}\right\}_{n=0}^{\infty} \subset\left(0, \min \left\{1, \frac{1}{L}\right\}\right)$ satisfies the condition $\lim _{n \rightarrow \infty}$ inf $\alpha_{n}>$ 0 , then the sequence $\left\{x^{n}\right\}_{n=0}^{\infty}$ generated by Algorithm 3.1 converges weakly to a fixed point of $T$.

Proof. For each $n \geq 0$, we have from (3.1)-(3.3) that

$$
x^{n+1}=\left(1-\alpha_{n}\right) x^{n}+\alpha_{n} T\left(x^{n+1}\right) .
$$

Consequently,

$$
x^{n}-x^{n+1}=\frac{\alpha_{n}}{1-\alpha_{n}}\left(x^{n+1}-T\left(x^{n+1}\right)\right) .
$$

For an arbitrary $x^{*} \in F i x(T)$, using (3.7), we get

$$
\begin{aligned}
\left\|x^{n+1}-x^{*}\right\|^{2}= & \left\|x^{n}-x^{*}-\frac{\alpha_{n}}{1-\alpha_{n}}\left(x^{n+1}-T\left(x^{n+1}\right)\right)\right\|^{2} \\
= & \left\|x^{n}-x^{*}\right\|^{2}+\frac{\alpha_{n}^{2}}{\left(1-\alpha_{n}\right)^{2}}\left\|x^{n+1}-T\left(x^{n+1}\right)\right\|^{2} \\
& +\frac{2 \alpha_{n}}{1-\alpha_{n}}\left\langle x^{*}-x^{n}, x^{n+1}-T\left(x^{n+1}\right)\right\rangle .
\end{aligned}
$$

From Lemma 2.1, we have that $I-T$ is monotone. Hence,

$$
\left\langle x^{*}-x^{n+1}, x^{n+1}-T\left(x^{n+1}\right)\right\rangle \leq\left\langle x^{*}-x^{n+1}, x^{*}-T\left(x^{*}\right)\right\rangle=0 .
$$

Using (3.7) again yields that

$$
\left\langle x^{n+1}-x^{n}, x^{n+1}-T\left(x^{n+1}\right)\right\rangle=-\frac{\alpha_{n}}{1-\alpha_{n}}\left\|x^{n+1}-T\left(x^{n+1}\right)\right\|^{2} .
$$

The combination of (3.8)-(3.10) leads to

$$
\left\|x^{n+1}-x^{*}\right\|^{2} \leq\left\|x^{n}-x^{*}\right\|^{2}-\frac{\alpha_{n}^{2}}{\left(1-\alpha_{n}\right)^{2}}\left\|x^{n+1}-T\left(x^{n+1}\right)\right\|^{2} .
$$

Thus we obtain from (3.11) that $\left\{\left\|x^{n}-x^{*}\right\|^{2}\right\}_{n=0}^{\infty}$ is nonincreasing, $\lim _{n \rightarrow \infty}\left\|x^{n}-x^{*}\right\|^{2}$ exists, and $\left\{x^{n}\right\}_{n=0}^{\infty}$ is bounded. From $\lim _{n \rightarrow \infty} \inf \alpha_{n}>0$, we also get from (3.11) that $\left\|x^{n}-T\left(x^{n}\right)\right\| \rightarrow 0$ as $n \rightarrow \infty$. 
Since $\left\{x^{n}\right\}_{n=0}^{\infty}$ is bounded, we have $\omega_{w}\left(x^{n}\right) \neq \emptyset$. Now we prove $\omega_{w}\left(x^{n}\right) \subset \operatorname{Fix}(T)$. For an arbitrary $\hat{x} \in \omega\left(x^{n}\right)$, there exists a subsequence $\left\{x^{n_{k}}\right\}_{k=0}^{\infty} \subset\left\{x^{n}\right\}_{n=0}^{\infty}$ such that $x^{n_{k}} \rightarrow \hat{x}$ as $k \rightarrow \infty$. Because $\|x-T(x)\|$ is a weakly lower semi-continuous function on $C$, we conclude that

$$
\|\hat{x}-T(\hat{x})\| \leq \lim _{k \rightarrow \infty}\left\|x^{n_{k}}-T\left(x^{n_{k}}\right)\right\|=0 .
$$

This means that $\hat{x} \in F i x(T)$. In order to complete the proof, all we need to do is to verify that $\omega_{w}\left(x^{n}\right)$ is a singleton. Indeed, for any $\hat{x}, \bar{x} \in \omega_{w}\left(x^{n}\right)$, there exist $\left\{x^{n_{k}}\right\}_{k=0}^{\infty}$ and $\left\{x^{n_{j}}\right\}_{j=0}^{\infty}$ such that $x^{n_{k}} \rightarrow \hat{x}$ and $x^{n_{j}} \rightarrow \bar{x}$, respectively. Since $\lim _{n \rightarrow \infty}\left\|x^{n}-\hat{x}\right\|^{2}$ and $\lim _{n \rightarrow \infty}\left\|x^{n}-\bar{x}\right\|^{2}$ exist, we have

$$
\begin{aligned}
\lim _{n \rightarrow \infty}\left\|x^{n}-\hat{x}\right\|^{2} & =\lim _{n \rightarrow \infty}\left\|x^{n}-\bar{x}+\bar{x}-\hat{x}\right\|^{2} \\
& =\lim _{j \rightarrow \infty}\left\{\left\|x^{n_{j}}-\bar{x}\right\|^{2}+2\left\langle x^{n_{j}}-\bar{x}, \bar{x}-\hat{x}\right\rangle+\|\bar{x}-\hat{x}\|^{2}\right\} \\
& =\lim _{j \rightarrow \infty}\left\|x^{n_{j}}-\bar{x}\right\|^{2}+\|\bar{x}-\hat{x}\|^{2} \\
& =\lim _{n \rightarrow \infty}\left\|x^{n}-\bar{x}\right\|^{2}+\|\bar{x}-\hat{x}\|^{2} .
\end{aligned}
$$

In the same way, we can obtain

$$
\lim _{n \rightarrow \infty}\left\|x^{n}-\bar{x}\right\|^{2}=\lim _{n \rightarrow \infty}\left\|x^{n}-\hat{x}\right\|^{2}+\|\bar{x}-\hat{x}\|^{2} .
$$

Combining (3.12) and (3.13), we assert $\|\hat{x}-\bar{x}\|=0$. Hence $\hat{x}=\bar{x}$.

Remark 3.2. Theorem 3.1 shows that the convergence of Algorithm 3.1 does not need the compactness assumption on $C$ or $T$, which is different from that of the Ishikawa iterative method (1.7).

Corollary 3.1. Let $C$ be a nonempty closed convex subset of $\mathscr{H}$ and let $T: C \rightarrow C$ be a nonexpansive mapping. If $\left\{\alpha_{n}\right\}_{n=0}^{\infty} \subset(0,1)$ satisfies the condition $\lim _{n \rightarrow \infty}$ inf $\alpha_{n}>0$, then the sequence $\left\{x^{n}\right\}_{n=0}^{\infty}$ generated by Algorithm 3.1 converges weakly to a fixed point of $T$.

Proof. From Lemma 2.1, we assert that $T$ is a pseudo-contractive mapping. Using Lemma 2.2, we have that $\|x-T(x)\|$ is a weakly lower semi-continuous function on $C$. Therefore, the desired result follows from Theorem 3.1 immediately.

Corollary 3.2. Let $C$ be a nonempty closed convex subset of $\mathscr{H}$ and let $T: C \rightarrow C$ be a L-Lipschitz continuous pseudo-contractive mapping. If $\mathscr{H}$ is finite dimensional and $\left\{\alpha_{n}\right\}_{n=0}^{\infty} \subset$ $\left(0, \min \left\{1, \frac{1}{L}\right\}\right)$ such that $\lim _{n \rightarrow \infty} \inf \alpha_{n}>0$, then the sequence $\left\{x^{n}\right\}_{n=0}^{\infty}$ generated by Algorithm 3.1 converges to a fixed point of $T$.

Proof. Because $T$ is Lipschitz continuous, it is obvious that $\|x-T(x)\|$ is continuous on $C$ and hence is also lower semi-continuous. On the other hand, since $\mathscr{H}$ is finite dimensional, we see that $\|x-T(x)\|$ is weaker semi-continuous. From Theorem 3.1, we can complete the proof immediately.

\section{THE INEXACT SUCCESSIVE CONTRACTION METHOD}

In this section, we turn to considering the inexact successive contraction method. In Algorithm 3.1, to solve subproblem (3.2) (i.e., to get $\bar{x}^{n+1}$ ), we need to use scheme (3.4). However, the actual calculation does not allow us to proceed the iteration process (3.4) infinitely. Therefore, we need to specify the iteration termination rule for iteration process (3.4) to ensure the 
convergence of the algorithm. To this end, we use a positive real control sequence $\left\{\varepsilon_{n}\right\}_{n=0}^{\infty}$ such that $\sum_{n=0}^{\infty} \varepsilon_{n}<+\infty$.

Algorithm 4.1. (The inexact successive contraction method)

Step 1: Choose $x^{0} \in C$ arbitrarily and set $n:=0$.

Step 2: For the current $x^{n}$, set $x_{0}^{n+1}=x^{n}$, choose $\alpha_{n}$ such that

$0<\alpha_{n}<\min \left\{1, \frac{1}{L}\right\}$, and calculate

$$
x_{m}^{n+1}=T_{n}\left(x_{m-1}^{n+1}\right), m=1, \ldots, m_{n},
$$

where $T_{n}$ is given by (3.1) and $m_{n}$ is the smallest positive integer such that

$$
\alpha_{n} \gamma_{n}^{m_{n}}\left\|x^{n}-T x^{n}\right\| \leq \varepsilon_{n}
$$

where $\gamma_{n}:=\alpha_{n} L$.

Step 3: Set

$$
x^{n+1}=x_{m_{n}}^{n+1}
$$

$n:=n+1$ and return to Step 2.

Theorem 4.1. Assume that the conditions of Theorem 3.1 are all satisfied. If $\sum_{n=0}^{\infty} \varepsilon_{n}<\infty$, then the sequence $\left\{x^{n}\right\}_{n=0}^{\infty}$ generated by Algorithm 4.1 converges weakly to a fixed point of $T$.

Proof. From (4.1), we get

$$
x_{m_{n}}^{n+1}=\left(1-\alpha_{n}\right) x^{n}+\alpha_{n} T x_{m_{n}-1}^{n+1},
$$

and consequently,

$$
x_{m_{n}}^{n+1}=x^{n}-\frac{\alpha_{n}}{1-\alpha_{n}}\left(x_{m_{n}}^{n+1}-T x_{m_{n}-1}^{n+1}\right) .
$$

For any $x^{*} \in F i x(T)$, we have from (4.5) that

$$
\begin{aligned}
\left\|x_{m_{n}}^{n+1}-x^{*}\right\|^{2}= & \left\|x^{n}-x^{*}-\frac{\alpha_{n}}{1-\alpha_{n}}\left(x_{m_{n}}^{n+1}-T x_{m_{n}-1}^{n+1}\right)\right\|^{2} \\
= & \left\|x^{n}-x^{*}\right\|^{2}+\frac{\alpha_{n}^{2}}{\left(1-\alpha_{n}\right)^{2}}\left\|x_{m_{n}}^{n+1}-T x_{m_{n}-1}^{n+1}\right\|^{2} \\
& +\frac{2 \alpha_{n}}{1-\alpha_{n}}\left\langle x^{*}-x^{n}, x_{m_{n}}^{n+1}-T x_{m_{n}-1}^{n+1}\right\rangle .
\end{aligned}
$$

It is obvious that

$$
\begin{aligned}
& \left\langle x^{*}-x^{n}, x_{m_{n}}^{n+1}-T x_{m_{n}-1}^{n+1}\right\rangle \\
= & \left\langle x^{*}-x_{m_{n}}^{n+1}, x_{m_{n}}^{n+1}-T x_{m_{n}}^{n+1}\right\rangle+\left\langle x^{*}-x_{m_{n}}^{n+1}, T x_{m_{n}}^{n+1}-T x_{m_{n}-1}^{n+1}\right\rangle \\
& +\left\langle x_{m_{n}}^{n+1}-x^{n}, x_{m_{n}}^{n+1}-T x_{m_{n}-1}^{n+1}\right\rangle .
\end{aligned}
$$

The monotonicity of $I-T$ leads to

$$
\left\langle x^{*}-x_{m_{n}}^{n+1}, x_{m_{n}}^{n+1}-T x_{m_{n}}^{n+1}\right\rangle \leq\left\langle x^{*}-x_{m_{n}}^{n+1}, x^{*}-T x^{*}\right\rangle=0 .
$$

Using (4.5), we have

$$
\left\langle x_{m_{n}}^{n+1}-x^{n}, x_{m_{n}}^{n+1}-T x_{m_{n}-1}^{n+1}\right\rangle=-\frac{\alpha_{n}}{\left(1-\alpha_{n}\right)}\left\|x_{m_{n}}^{n+1}-T x_{m_{n}-1}^{n+1}\right\|^{2} .
$$


Note that $T$ is $L$-Lipschitz continuous, $T_{n}$ is a strict contraction with the coefficient $\gamma_{n}=\alpha_{n} L$, and $x_{0}^{n+1}=x^{n}$, we have

$$
\begin{aligned}
\left\langle x^{*}-x_{m_{n}}^{n+1}, T x_{m_{n}}^{n+1}-T x_{m_{n}-1}^{n+1}\right\rangle & \leq\left\|x^{*}-x_{m_{n}}^{n+1}\right\|\left\|T x_{m_{n}}^{n+1}-T x_{m_{n}-1}^{n+1}\right\| \\
& \leq L\left\|x^{*}-x_{m_{n}}^{n+1}\right\|\left\|x_{m_{n}}^{n+1}-x_{m_{n}-1}^{n+1}\right\| \\
& \leq L \gamma_{n}^{m_{n}-1}\left\|x^{*}-x_{m_{n}}^{n+1}\right\|\left\|x_{1}^{n+1}-x_{0}^{n+1}\right\| \\
& =\gamma_{n}^{m_{n}}\left\|x^{*}-x_{m_{n}}^{n+1}\right\|\left\|x^{n}-T x^{n}\right\| .
\end{aligned}
$$

Using (4.6)-(4.10), we obtain

$$
\begin{aligned}
\left\|x_{m_{n}}^{n+1}-x^{*}\right\|^{2} \leq & \left\|x^{n}-x^{*}\right\|^{2}-\frac{\alpha_{n}^{2}}{\left(1-\alpha_{n}\right)^{2}}\left\|x_{m_{n}}^{n+1}-T x_{m_{n}-1}^{n+1}\right\|^{2} \\
& +\frac{2 \alpha_{n} \gamma_{n}^{m_{n}}}{1-\alpha_{n}}\left\|x^{*}-x_{m_{n}}^{n+1}\right\|\left\|x^{n}-T x^{n}\right\| .
\end{aligned}
$$

On the other hand, we deduce

$$
\begin{aligned}
\left\|x_{m_{n}}^{n+1}-T x_{m_{n}-1}^{n+1}\right\| & =\left\|x_{m_{n}}^{n+1}-T x_{m_{n}}^{n+1}+T x_{m_{n}}^{n+1}-T x_{m_{n}-1}^{n+1}\right\| \\
& \geq\left\|x_{m_{n}}^{n+1}-T x_{m_{n}}^{n+1}\right\|-\left\|T x_{m_{n}}^{n+1}-T x_{m_{n}-1}^{n+1}\right\|,
\end{aligned}
$$

and

$$
\begin{aligned}
-\left\|x_{m_{n}}^{n+1}-T x_{m_{n}-1}^{n+1}\right\|^{2} \leq & -\left\|x_{m_{n}}^{n+1}-T x_{m_{n}}^{n+1}\right\|^{2}-\left\|T x_{m_{n}}^{n+1}-T x_{m_{n}-1}^{n+1}\right\|^{2} \\
& +2\left\|x_{m_{n}}^{n+1}-T x_{m_{n}}^{n+1}\right\|\left\|T x_{m_{n}}^{n+1}-T x_{m_{n}-1}^{n+1}\right\| \\
\leq & -\left\|x_{m_{n}}^{n+1}-T x_{m_{n}}^{n+1}\right\|^{2}-\left\|T x_{m_{n}}^{n+1}-T x_{m_{n}-1}^{n+1}\right\|^{2} \\
& +\frac{1}{4}\left\|x_{m_{n}}^{n+1}-T x_{m_{n}}^{n+1}\right\|^{2}+4\left\|T x_{m_{n}}^{n+1}-T x_{m_{n}-1}^{n+1}\right\|^{2} \\
\leq & -\frac{3}{4}\left\|x_{m_{n}}^{n+1}-T x_{m_{n}}^{n+1}\right\|^{2}+3\left\|T x_{m_{n}}^{n+1}-T x_{m_{n}-1}^{n+1}\right\|^{2} .
\end{aligned}
$$

It follows from (4.11) and (4.12) that

$$
\begin{aligned}
& \left\|x_{m_{n}}^{n+1}-x^{*}\right\|^{2} \\
\leq & \left\|x^{n}-x^{*}\right\|^{2}-\frac{3 \alpha_{n}^{2}}{4\left(1-\alpha_{n}\right)^{2}}\left\|x_{m_{n}}^{n+1}-T x_{m_{n}}^{n+1}\right\|^{2}+\frac{3 \alpha_{n}^{2}}{\left(1-\alpha_{n}\right)^{2}}\left\|T x_{m_{n}}^{n+1}-T x_{m_{n}-1}^{n+1}\right\|^{2} \\
& +\frac{2 \alpha_{n} \gamma_{n}^{m_{n}}}{1-\alpha_{n}}\left\|x^{*}-x_{m_{n}}^{n+1}\right\|\left\|x^{n}-T x^{n}\right\| \\
\leq & \left\|x^{n}-x^{*}\right\|^{2}-\frac{3 \alpha_{n}^{2}}{4\left(1-\alpha_{n}\right)^{2}}\left\|x_{m_{n}}^{n+1}-T x_{m_{n}}^{n+1}\right\|^{2}+\frac{3 \alpha_{n}^{2} \gamma_{n}^{2 m_{n}}}{\left(1-\alpha_{n}\right)^{2}}\left\|x^{n}-T x^{n}\right\|^{2} \\
& +\alpha_{n} \gamma_{n}^{m_{n}}\left\|x^{*}-x_{m_{n}}^{n+1}\right\|^{2}\left\|x^{n}-T x^{n}\right\|+\frac{\alpha_{n} \gamma_{n}^{m_{n}}}{\left(1-\alpha_{n}\right)^{2}}\left\|x^{n}-T x^{n}\right\| .
\end{aligned}
$$

Setting $\sigma_{n}=\alpha_{n} \gamma_{n}^{m_{n}}\left\|x^{n}-T x^{n}\right\|$ and using $x^{n+1}=x_{m_{n}}^{n+1}$, we have that (4.13) can be rewritten as the following form

$$
\begin{aligned}
\left\|x^{n+1}-x^{*}\right\|^{2} \leq & \left(1+\frac{\sigma_{n}}{1-\sigma_{n}}\right)\left\|x^{n}-x^{*}\right\|^{2}-\frac{3 \alpha_{n}^{2}}{4\left(1-\sigma_{n}\right)\left(1-\alpha_{n}\right)^{2}}\left\|x^{n+1}-T x^{n+1}\right\|^{2} \\
& +\frac{\left(3 \sigma_{n}+1\right) \sigma_{n}}{\left(1-\alpha_{n}\right)^{2}\left(1-\sigma_{n}\right)} .
\end{aligned}
$$


From (4.2) and the condition $\sum_{n=0}^{\infty} \varepsilon_{n}<+\infty$, it is easy to see that

$$
\sum_{n=0}^{\infty} \frac{\sigma_{n}}{1-\sigma_{n}}<+\infty
$$

and

$$
\sum_{n=0}^{\infty} \frac{\left(3 \sigma_{n}+1\right) \sigma_{n}}{\left(1-\alpha_{n}\right)^{2}\left(1-\sigma_{n}\right)}<+\infty
$$

By applying Lemma 2.3 to (4.14), we assert that $\lim _{n \rightarrow \infty}\left\|x^{n}-x^{*}\right\|^{2}$ exists. Hence, $\left\{x^{n}\right\}_{n=0}^{\infty}$ is bounded. This together with (4.14) implies that $\left\|x^{n+1}-T\left(x^{n+1}\right)\right\| \rightarrow 0$ as $n \rightarrow \infty$. Furthermore, by repeating the argument of the final part of the proof of Theorem 3.1, we also have that $\omega_{w}\left(x^{n}\right) \subset \operatorname{Fix}(T)$ and the sequence $\left\{x^{n}\right\}_{n=0}^{\infty}$ converges weakly to a fixed point of $T$.

Similar to Corollary 3.1 and Corollary 3.2, the following conclusions can be easily obtained and hence their proofs are omitted.

Corollary 4.1. Let $C$ be a nonempty closed convex subset of $\mathscr{H}$ and let $T: C \rightarrow C$ be a nonexpansive mapping. If $\left\{\alpha_{n}\right\}_{n=0}^{\infty} \subset(0,1)$ and $\left\{\varepsilon_{n}\right\}_{n=0}^{\infty}$ satisfy $\lim _{n \rightarrow \infty} \inf \alpha_{n}>0$ and $\sum_{n=0}^{\infty} \varepsilon_{n}<\infty$, respectively, then the sequence $\left\{x^{n}\right\}_{n=0}^{\infty}$ generated by Algorithm 4.1 converges weakly to a fixed point of $T$.

Corollary 4.2. Let $C$ be a nonempty closed convex subset of a finite dimensional Hilbert space $\mathscr{H}$ and let $T: C \rightarrow C$ be a L-Lipschitz continuous pseudo-contractive mapping. If $\left\{\alpha_{n}\right\}_{n=0}^{\infty} \subset$ $\left(0, \min \left\{1, \frac{1}{L}\right\}\right)$ and $\left\{\varepsilon_{n}\right\}_{n=0}^{\infty}$ satisfy $\lim _{n \rightarrow \infty} \inf \alpha_{n}>0$ and $\sum_{n=0}^{\infty} \varepsilon_{n}<\infty$, respectively, , then the sequence $\left\{x^{n}\right\}_{n=0}^{\infty}$ generated by Algorithm 4.1 converges to a fixed point of $T$.

At the end of this section, we compare the effectiveness of Algorithm 4.1 and Ishikawa iteration process (1.7) by analyzing a simple example as follows.

Example 4.1. Let $\mathscr{H}=\mathbb{R}^{2}$ and let $T: \mathbb{R}^{2} \rightarrow \mathbb{R}^{2}$ be a bounded linear operator defined by the formula:

$$
T\left(\begin{array}{l}
\xi \\
\eta
\end{array}\right)=\left(\begin{array}{rr}
1 & -1 \\
1 & 1
\end{array}\right)\left(\begin{array}{l}
\xi \\
\eta
\end{array}\right), \forall\left(\begin{array}{l}
\xi \\
\eta
\end{array}\right) \in \mathbb{R}^{2} .
$$

Obviously, $(0,0)^{\top}$ is the unique fixed point of $T$. From (4.15), we have $\langle x, T x\rangle=\|x\|^{2}$ holds for any $x \in \mathbb{R}^{2}$. With the help of Lemma 2.1, this implies that $T$ is a pseudo-contractive mapping. Since $\|T x\|=\sqrt{2}\|x\|, \forall x \in \mathbb{R}^{2}$, we have $\|T\|=\sqrt{2}$.

In Ishikawa iteration process (1.7), the parameter sequence $\left\{\alpha_{n}\right\}_{n=0}^{\infty}$ and $\left\{\beta_{n}\right\}_{n=0}^{\infty}$ are set to be $\alpha_{n}=\beta_{n}=\frac{1}{\sqrt{n+1}}$ for all $n \geq 0$ according to Theorem 1.2. However, it is worth pointing out that the convergence of the Ishikawa iterative process can not be guaranteed because Theorem 1.2 is invalid for this example. Indeed, $T$ cannot be regarded as a self-mapping defined on any bounded closed convex set $C \subset \mathbb{R}^{2}$ (unless $C$ is set to be $C=\left\{(0,0)^{\top}\right\}$ ), because, for any non-zero vector $u \in \mathbb{R}^{2},\left\|T^{n}(u)\right\|=(\sqrt{2})^{n}\|u\| \rightarrow \infty$ as $n \rightarrow \infty$. In other words, the compactness condition on the domain of $T$ in Theorem 1.2 is not satisfied.

It is easy to see that this example can be easily solved by using Algorithm 4.1. In fact, the sequence $\left\{x^{n}\right\}_{n=0}^{\infty}$ generated by Algorithm 4.1 converges to the unique fixed point $(0,0)^{\top}$ of $T$ as long as the conditions on $\left\{\alpha_{n}\right\}_{n=0}^{\infty}$ and $\left\{\varepsilon_{n}\right\}_{n=0}^{\infty}$ in Corollary 4.2 are satisfied. In Algorithm 
4.1, the parameter sequence $\left\{\alpha_{n}\right\}_{n=0}^{\infty}$ and the control sequence $\left\{\varepsilon_{n}\right\}_{n=0}^{\infty}$ are set to be $\alpha_{n}=0.6$ and $\varepsilon_{n}=\frac{1}{2^{n+1}}$ for all $n \geq 0$, respectively.

We use $E_{n}=\left\|x^{n}\right\|$ to measure the error of the $n$-th step iteration. The stopping criteria is that $E_{n} \leq \varepsilon$, where $\varepsilon$ is the given required precision. The numerical results are list in Table I below. In Table 1, Iter. $(n)$ denotes the number of iterations and CPU(s) denotes the computing time in seconds. From Table 1, it concludes that Algorithm 4.1 is more effective than Ishikawa iterative method (1.7).

Table 1: Comparison of Algorithm 4.1 and Ishikawa iterative method (1.7).

\begin{tabular}{|c|c|c|c|c|c|}
\hline \multirow{2}{*}{$\varepsilon$} & \multicolumn{3}{|c|}{ Algorithm 4.1 } & \multicolumn{2}{c|}{ Ishikawa iteration } \\
\cline { 2 - 6 } & Iter. $(n)$ & $m_{n}$ & CPU (s) & Iter. $(n)$ & CPU (s) \\
\hline \hline $10^{-3}$ & 11 & 1 & 1.7378 & 29637 & 0.1707 \\
\hline $10^{-5}$ & 19 & 4 & 3.0746 & 2979276 & 10.7221 \\
\hline $10^{-7}$ & 24 & 1 & 3.6133 & $\ldots \ldots$ & $\ldots \ldots$ \\
\hline $10^{-9}$ & 32 & 1 & 4.7684 & $\ldots \ldots$ & $\cdots \cdots$ \\
\hline $10^{-11}$ & 39 & 5 & 6.2492 & $\ldots \ldots$ & $\ldots \ldots$ \\
\hline
\end{tabular}

\section{Acknowledgments}

The authors thanks the anonymous reviewers for useful suggestions which improved this paper. This paper was supported by Scientific Research Project of Tianjin Municipal Education Commission (No. 2018KJ253).

\section{REFERENCES}

[1] G.L. Acedo, H.K. Xu, Iterative methods for strict pseudo-contractions in Hilbert spaces, Nonlinear Anal. 67 (2007), 2258-2271.

[2] H.H. Bauschke, P.L. Combettes, Convex analysis and monotone operator theory in Hilbert spaces, Springer, New York, 2010.

[3] C.L. Byrne, Iterative Optimization in Inverse Problems, CRC Press, Boca Raton, 2014.

[4] Q. Cheng, Y. Su, J. Zhang, Convergence theorems of a three-step iteration method for a countable family of pseudocontractive mappings, Fixed Point Theory Appl. 2013 (2013), 1-14.

[5] C.E. Chidume, S.A. Mutangadura, An example of the Mann iteration method for Lipschitz pseudocontractions, Proc. Amer. Math. Soc. 129 (2001), 2359-2363.

[6] C.E. Chidume, C. Moore, The solution by iteration of nonlinear equations in uniformly smooth Banach spaces, J. Math. Anal. Appl. 215 (1997), 132-146.

[7] C.E. Chidume, C. Moore, Fixed point iteration for pseudocontractive maps, Proc. Amer. Math. Soc. 127 (1999), 1163-1170.

[8] K. Goebel, S. Reich, Uniform Convexity, Hyperbolic Geometry and Nonexpansive Mappings, Marcel Dekker, New York and Basel 1984.

[9] B. Halpern, Fixed points of nonexpanding maps, Bull. Amer. Math. Soc. 73 (1967), 957-961.

[10] S. He, H. Tian, Selective projection methods for solving a class of variational inequalities, Numer. Algor. 80 (2019) 617-634.

[11] S. He, H.K. Xu, Variational inequalities governed by boundedly Lipschitzian and strongly monotone operators, Fixed Point Theory, 10 (2009), 245-258.

[12] S. He, H. Tian, H.K. Xu, The selective projection method for convex feasibility and split feasibility problems, J. Nonlinear Convex Anal. 19 (2018), 1199-1215.

[13] S. Ishikawa, Fixed points by a new iteration method, Proc. Amer. Math. Soc. 44 (1974), 147-150. 
[14] L.S. Liu, Ishikawa and Mann iterative processes with errors for nonlinear strongly accretive mapping in Banach spaces, J. Math. Anal. Appl. 194 (1995), 114-125.

[15] W.R. Mann, Mean value methods in iteration, Proc. Amer. Math. Soc. 4 (1953), 506-510.

[16] Z. Opial, Weak convergence of the sequence of successive approximations of nonexpansive mappings, Bull. Amer. Math. Soc. 73 (1967), 595-597.

[17] X. Qin, S.Y. Cho, L. Wang, Iterative algorithms with errors for zero points of $\mathrm{m}$-accretive operators, Fixed Point Theory Appl. 2013 (2013), Article ID 148.

[18] H.K. Xu, Iterative algorithm for nonlinear operators, J. London Math. Soc. 66 (2002), 240-256.

[19] H. Zegeye, N. Shahzad, M.A. Alghamdi, Convergence of Ishikawa's iteration method for pseudocontractive mappings, Nonlinear Anal. 74 (2011), 7304-7311.

[20] H. Zhou, Convergence theorems of fixed points for Lipschitz pseudo-contractions in Hilbert spaces, J. Math. Anal. Appl. 343 (2008), 546-556. 